\title{
Article \\ Photosensitive Bent-Core Liquid Crystals with Laterally Substituted Azobenzene Unit
}

\author{
Diana Jágerová ${ }^{1,+}$, Michal Šmahel ${ }^{1,+}$, Anna Poryvai ${ }^{1}\left(\mathbb{D}\right.$, Jan $_{\text {Macháček }}^{2}\left(\mathbb{D}\right.$, Vladimíra Novotná ${ }^{3}(\mathbb{D}$ \\ and Michal Kohout $1, *$ (D)
}

1 Department of Organic Chemistry, University of Chemistry and Technology, CZ-166 28 Prague, Czech Republic; jagerovd@vscht.cz (D.J.); smahelm@vscht.cz (M.Š.); poryvaia@vscht.cz (A.P.)

2 Department of Glass and Ceramics, University of Chemistry and Technology, CZ-166 28 Prague, Czech Republic; Jan.Machacek@vscht.cz

3 Institute of Physics of the Czech Academy of Sciences, Na Slovance 2, CZ-182 21 Prague, Czech Republic; novotna@fzu.cz

* Correspondence: michal.kohout@vscht.cz; Tel.: +420-220443688

+ Both authors contributed equally to the work.

Citation: Jágerová, D.; Šmahel, M.; Poryvai, A.; Macháček, J.; Novotná,

V.; Kohout, M. Photosensitive Bent-Core Liquid Crystals with Laterally Substituted Azobenzene Unit. Crystals 2021, 11, 1265. https:// doi.org/10.3390/cryst11101265

Academic Editors: Ingo Dierking and M. Blanca Ros

Received: 30 September 2021

Accepted: 16 October 2021

Published: 18 October 2021

Publisher's Note: MDPI stays neutral with regard to jurisdictional claims in published maps and institutional affiliations.

Copyright: (c) 2021 by the authors. Licensee MDPI, Basel, Switzerland. This article is an open access article distributed under the terms and conditions of the Creative Commons Attribution (CC BY) license (https:// creativecommons.org/licenses/by/ $4.0 /)$.

\begin{abstract}
Photosensitive liquid crystals represent an important class of functional materials that experience rapid development. Hereby, we present novel bent-core liquid crystals bearing a lateral substitution on the central core and in the vicinity of the photosensitive unit-an azo group. The azo group enables fast (E)-to-(Z)-isomerization upon irradiation with UV-light and visible light, while the substitution facilitates the high stability of the photochemically formed ( $Z$ )-isomer. The effectiveness of the irradiation and the composition of photostationary states was determined by UV/Vis and ${ }^{1} \mathrm{H}$ NMR spectroscopy. A nematic phase formed by the materials was characterized by differential scanning calorimetry and optical polarizing microscopy. We show that the materials easily change their relative configuration of the $\mathrm{N}=\mathrm{N}$ double bond not only in solution, but also in the mesophase, which leads to fast isothermal phase transition from the nematic phase to isotropic liquid.
\end{abstract}

Keywords: photosensitive liquid crystals; azobenzene; bent-core liquid crystals; substituted azobenzene; stable (Z)-isomer; mesomorphic properties; photostationary state; NMR spectroscopy

\section{Introduction}

Thermotropic liquid crystals (LCs) are well-known for their unique ability to selfassemble and form long-range ordered structures, so-called mesophases between anisotropic solid and isotropic liquid states. Various mesophases can be easily tuned by external stimuli such as temperature, mechanical pressure, and magnetic or electric fields. Thanks to the combination of fluidity and anisotropy of optical and optoelectrical properties, they are used on daily basis, e.g., in liquid crystal displays (LCDs), optical shutters or contact thermometers [1]. In the last decade, photo responsive liquid-crystalline materials, whose properties can be modulated by ultraviolet (UV) or visible light, have been intensively studied due to their possible applications in various photonic devices [2,3], optical memories [4], optical switches [5], displays or artificial muscles [6,7].

From the pool of feasible photosensitive units, the azobenzene moiety is the most frequently utilized motive in the structure of LCs to induce photo-responsivity [8]. (E)-(Z) isomerization, triggered by an external light source of a specific wavelength, results in changes of geometry and thus breaks the supramolecular arrangement within a mesophase. This typically leads to the decrease of order parameter of the system and the transition from a mesophase into the isotropic liquid, followed by visible light-triggered and/or thermal relaxation into the original state [9-11]. From this point of view, the rate of the $(E)-(Z)$ photoisomerization and thermal back $(Z)-(E)$ relaxation are two important parameters for real applications. Photoswitches with very fast or very slow relaxation, on the other 
hand, are both desired for different types of applications (e.g., optical storage devices). Various lateral substitution neighbouring the azo group can modulate the mesomorphic and photochromic properties $[12,13]$ of formed mesophases $[14,15]$ and, thus, can be used to fine-tune the materials properties. Apart from that, asymmetric azobenzene materials with different terminal alkyl chains can be used to prepare materials with room temperature mesophases. In a similar manner, multicomponent mixtures containing azobenzene-based LCs can be utilized [16]. The properties of such materials can be then modulated by low power UV and/or visible light, which gives rise to optically controled spatial solitons or the formation of holographic gratings [17,18].

Bent-core liquid crystals (BCLCs) are an extraordinary class of the mesomorphic materials since they may exhibit polar order and macroscopic chirality in self-assembled mesophases consisting of achiral molecules. According to their structure, they can form various mesophases with unique properties in comparison with other classes of LCs [19-21]. Bent-core nematics $(\mathrm{BCN})$ can have biaxial character [22-26] and exhibit a huge flexoelectric effect compared to conventional calamitic nematics [27-30], which predetermines them for new generation of optoelectrical devices, holographic media [31,32], photonics and optical data storage [33,34]. In this study, we focused on a group of BCLCs possessing the azo group in one of the elongating side arms. With the aim of preferably preparing $\mathrm{BCN}$ materials, we introduced a lateral substituent (fluorine) into the central core. This substitution, together with the orientation of the ester linking units, previously led to the preferential formation of a nematic phase [35]. Lateral substituents in the azobenzene part of the structure should pose a strong effect on the stability of photochemically formed (Z)-isomers. This effect was already described for calamitic materials [14], but has not been studied for BCLCs to date.

\section{Materials and Methods}

\subsection{Design and Synthesis of Materials}

Encouraged by our recent investigation on light-tunable flexoelectric domains [2], we have designed a new series of BCLCs (Ia-d) with a different substitution neighboring the azo group (Figure 1) to modulate the photochemical properties.<smiles>CCCCCOc1ccc(N=Nc2ccc(C(=O)Oc3cc(C(=O)Oc4ccc(OC(=O)c5ccc(OCCCC)cc5)cc4)ccc3F)cc2)cc1</smiles>



Figure 1. (a) Previously investigated material SV775, [2] and (b) structure of the new material Ia-d.

The designed materials were prepared from two separately synthesized building blocks (Figure 2). Photosensitive arms (4a-d) were connected via acylation with phenol (7) according to the previously published procedure [2] to afford the final bent-core molecules (Ia-d). 


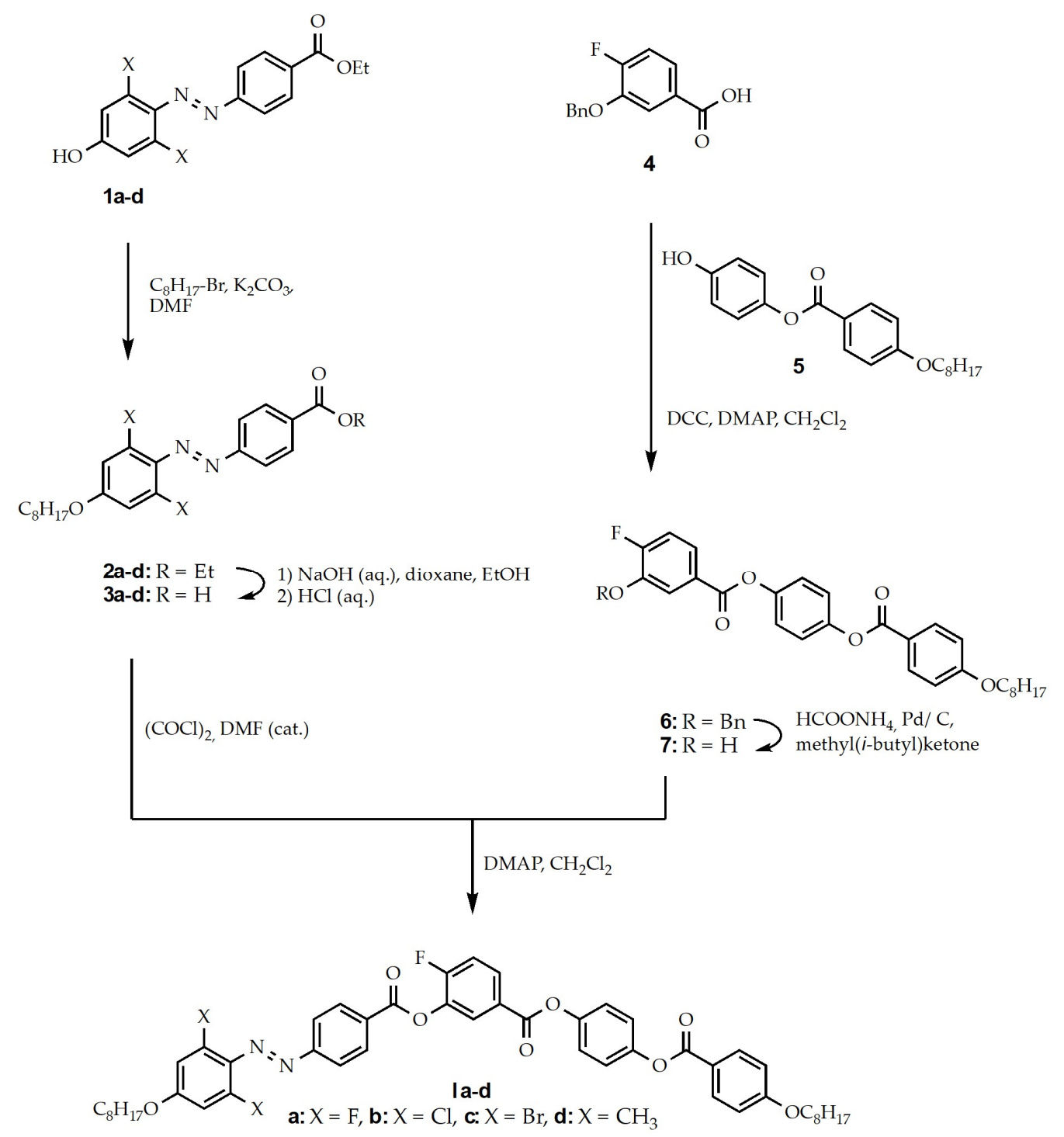

Figure 2. Synthesis of the final materials Ia-d.

\subsection{Synthesis and Characterization}

All commercially available reagents were purchased from Merck (Darmstadt, Germany) and TCI Chemicals Europe (Zwijndrecht, Belgium) and used without further purification. Photosensitive precursors 1a-d, fluorinated acid 4 and phenol 5 were prepared previously, according to known procedure, and their analytical data correspond to that described in the literature [14]. Dry dichloromethane and dimethylformamide (DMF) were taken from the solvent purification system PureSolv MD7 (Amesbury, MA, USA). For column chromatography, a Kieselgel 60-100 $\mu \mathrm{m}$ (Merck, Darmstadt, Germany) was used.

The structures of the intermediates and final products were confirmed by ${ }^{1} \mathrm{H}$ and ${ }^{13}$ C NMR spectroscopy. Spectra were acquired on an Agilent 400-MR DDR2 spectrometer (Agilent Technologies, Santa Clara, CA, USA), chemical shifts $(\delta)$ are given in ppm, and spinspin coupling constants $(J)$ in $\mathrm{Hz}$. Deuterated chloroform $\left(\mathrm{CDCl}_{3}\right)$ and dimethylsulfoxide (d6-DMSO) were used as solvents. The residual non-deuterated peak was used as the internal standard for the NMR spectra. The multiplicity of recorded peaks is described using following abbreviations: $\mathrm{d}$-doublet, $\mathrm{t}$-triplet, $\mathrm{q}$-quartet, $\mathrm{m}$-multiplet. Three general methods (A-alkylation, B — ester hydrolysis and C-acylation with acid chloride) were used for the preparation of all final BCLCs.

General method A. To a stirred solution of phenol (1.0 eq.) and freshly dried potassium carbonate (1.1 eq.) in dry DMF $(10 \mathrm{~mL} / \mathrm{mmol})$, octylbromide (1.1 eq.) was added and the 
reaction mixture was heated to reflux for $2 \mathrm{~h}$ in an inert atmosphere. Full conversion of reagents was detected by TLC (eluent toluene:MTBE, 30:1). After cooling to room temperature, the reaction mixture was decomposed with water $(50 \mathrm{~mL} / \mathrm{mmol})$ and extracted with toluene $(3 \times 50 \mathrm{~mL} / \mathrm{mmol})$. The collected organic solution was dried with anhydrous $\mathrm{MgSO}_{4}$. After filtration, the solvent was removed under reduced pressure and the crude product was purified by column chromatography (eluent toluene:MTBE, 40:1).

General method B. To a stirred solution of ester (1 eq.) in dioxane $(30 \mathrm{~mL} / \mathrm{mmol})$, ethanol (30 mL/mmol) and aqueous solution, $0.5 \mathrm{M}$ of natrium hydroxide (3 eq.) was added and the reaction mixture was heated to reflux for $1.5 \mathrm{~h}$. After cooling to room temperature, EtOH was removed under reduced pressure and the resulting suspension was diluted with water $(20 \mathrm{~mL} / \mathrm{mmol})$ and acidified with $\mathrm{HCl}(17 \%)$ to $\mathrm{pH}$ approx. 1-2. The precipitate was filtered out, dried under reduced pressure, and used in the following reaction without further purification.

General method C. To a stirred suspension of acid (1.0 eq.) in oxalyl chloride $(10 \mathrm{~mL} / \mathrm{mmol})$ a catalytic amount of DMF $(0.05 \mathrm{~mL})$ was added. The resulting mixture was heated to reflux, and after $45 \mathrm{~min}$ the excess oxalyl chloride was distilled off. The residue was dissolved in hexane $(10 \mathrm{~mL} / \mathrm{mmol})$ and treated with active charcoal at reflux. After the mixture was filtered while hot, the hexane was evaporated, and the crude acid chloride was mixed with a solution of DMAP (1.1 eq.) and phenol (1 eq.) in dry toluene $(15 \mathrm{~mL} / \mathrm{mmol})$. The mixture was stirred in an argon atmosphere at room temperature for $8 \mathrm{~h}$, and then decomposed with $17 \%$ aq. hydrochloric acid. The layers were separated, and the aqueous layer was extracted with chloroform $(2 \times 30 \mathrm{~mL} / \mathrm{mmol})$. The combined organic solution was dried with anhydrous $\mathrm{MgSO}_{4}$. The solvent was removed and the crude orange product was purified by column chromatography (eluent toluene:MTBE, 30:1) and multiple crystallizations from the EtOH/ EtOAc (1:1) mixture.

\section{Ethyl (E)-4-\{[2,6-difluoro-4-(octyloxy)phenyl]diazenyl\}benzoate (2a)}

According to the general method $\mathrm{A}$, the intermediate $2 \mathrm{a}$ was prepared and purified to yield $0.97 \mathrm{~g}(71 \%)$ of orange solid, m.p. $108-111^{\circ} \mathrm{C} .{ }^{1} \mathrm{H} \mathrm{NMR}\left(400 \mathrm{MHz}, \mathrm{CDCl}_{3}\right): 0.89(\mathrm{t}$, $\left.3 \mathrm{H}, \mathrm{J}=7.1, \mathrm{CH}_{3}\right), 1.25-1.51\left(\mathrm{~m}, 10 \mathrm{H}, 5 \times \mathrm{CH}_{2}\right), 1.42\left(\mathrm{t}, 3 \mathrm{H}, J=7.1, \mathrm{CH}_{3}\right), 1.77-1.84(\mathrm{~m}, 2 \mathrm{H}$, $\left.\mathrm{CH}_{2}\right), 4.01\left(\mathrm{t}, 2 \mathrm{H}, J=6.5, \mathrm{OCH}_{2}\right), 4.41\left(\mathrm{q}, 2 \mathrm{H}, J=7.1, \mathrm{OCH}_{2}\right), 6.59(\mathrm{~d}, 2 \mathrm{H}, J=11.2, \mathrm{Ar}), 7.90$ $(\mathrm{d}, 2 \mathrm{H}, J=8.6, \mathrm{Ar}), 8.17(\mathrm{~d}, 2 \mathrm{H}, J=8.6, \mathrm{Ar}){ }^{13} \mathrm{C} \mathrm{NMR}\left(100 \mathrm{MHz}, \mathrm{CDCl}_{3}\right): 14.10\left(\mathrm{CH}_{3}\right), 14.33$ $\left(\mathrm{CH}_{3}\right), 22.65\left(\mathrm{CH}_{2}\right), 25.87\left(\mathrm{CH}_{2}\right), 28.84\left(\mathrm{CH}_{2}\right), 29.18\left(\mathrm{CH}_{2}\right), 29.25\left(\mathrm{CH}_{2}\right), 31.77\left(\mathrm{CH}_{2}\right), 61.25$ $\left(\mathrm{OCH}_{2}\right), 69.18\left(\mathrm{OCH}_{2}\right), 99.30\left(\mathrm{dd}, 2 \times \mathrm{CH}-\mathrm{Ar}, J^{\mathrm{C}-\mathrm{F}}=24.0,3.0\right), 122.32(2 \times \mathrm{CH}-\mathrm{Ar}), 125.17(\mathrm{t}$, $\left.\mathrm{C}-\mathrm{Ar}, J^{\mathrm{C}-\mathrm{F}}=9.3\right), 130.52(2 \times \mathrm{CH}-\mathrm{Ar}), 131.12(\mathrm{C}-\mathrm{Ar}), 155.96(\mathrm{C}-\mathrm{Ar}), 157.70(\mathrm{dd}, 2 \times \mathrm{CH}-\mathrm{Ar}$, $\left.J^{C-F}=261.0,7.3\right), 162.00\left(\mathrm{t}, \mathrm{C}-\mathrm{Ar}, J^{C-F}=14.0\right), 166.08(\mathrm{C}=\mathrm{O})$.

\section{Ethyl (E)-4-\{[2,6-dichloro-4-(octyloxy)phenyl]diazenyl\}benzoate (2b)}

According to the general method $\mathrm{A}$, the intermediate $2 \mathrm{~b}$ was prepared and purified to yield $1.12 \mathrm{~g}(84 \%)$ of orange solid, m.p. $55-58{ }^{\circ} \mathrm{C} .{ }^{1} \mathrm{H} \mathrm{NMR}\left(400 \mathrm{MHz}, \mathrm{CDCl}_{3}\right): 0.88(\mathrm{t}, 3 \mathrm{H}$, $\left.J=7.1, \mathrm{CH}_{3}\right), 1.22-1.48\left(\mathrm{~m}, 10 \mathrm{H}, 5 \times \mathrm{CH}_{2}\right), 1.43\left(\mathrm{t}, 3 \mathrm{H}, J=7.1, \mathrm{CH}_{3}\right), 1.76-1.84\left(\mathrm{~m}, 2 \mathrm{H}, \mathrm{CH}_{2}\right)$, $3.99\left(\mathrm{t}, 2 \mathrm{H}, \mathrm{J}=6.6, \mathrm{OCH}_{2}\right), 4.42\left(\mathrm{q}, 2 \mathrm{H}, J=7.1, \mathrm{OCH}_{2}\right), 6.98(\mathrm{~s}, 2 \mathrm{H}, \mathrm{Ar}), 7.96(\mathrm{~d}, 2 \mathrm{H}, J=8.9$, $\mathrm{Ar}), 8.21(\mathrm{~d}, 2 \mathrm{H}, \mathrm{J}=8.9, \mathrm{Ar}) .{ }^{13} \mathrm{C} \mathrm{NMR}\left(100 \mathrm{MHz}, \mathrm{CDCl}_{3}\right): 14.11\left(\mathrm{CH}_{3}\right), 14.32\left(\mathrm{CH}_{3}\right), 22.65$ $\left(\mathrm{CH}_{2}\right), 25.89\left(\mathrm{CH}_{2}\right), 28.94\left(\mathrm{CH}_{2}\right), 29.20\left(\mathrm{CH}_{2}\right), 29.26\left(\mathrm{CH}_{2}\right), 31.78\left(\mathrm{CH}_{2}\right), 61.34\left(\mathrm{OCH}_{2}\right), 68.96$ $\left(\mathrm{OCH}_{2}\right), 115.67$ (2× CH-Ar), 122.63 (2× CH-Ar), 129.97 (C-Ar), 130.60 (2× CH-Ar), 132.79 (C-Ar), 141.82 (C-Ar), 157.32 (C-Ar), 159.06 (2× C-Ar), 165.95 (C=O).

\section{Ethyl (E)-4-\{[2,6-dibromo-4-(octyloxy)phenyl]diazenyl\}benzoate (2c)}

According to the general method $\mathrm{A}$, the intermediate $2 \mathrm{c}$ was prepared and purified to yield $1.04 \mathrm{~g}(83 \%)$ of orange solid, m.p. $53-56{ }^{\circ} \mathrm{C} .{ }^{1} \mathrm{H} \mathrm{NMR}\left(400 \mathrm{MHz}, \mathrm{CDCl}_{3}\right): 0.90(\mathrm{t}, 3 \mathrm{H}$, $\left.J=6.8, \mathrm{CH}_{3}\right), 1.23-1.53\left(\mathrm{~m}, 10 \mathrm{H}, 5 \times \mathrm{CH}_{2}\right), 1.43\left(\mathrm{t}, 3 \mathrm{H}, J=7.1, \mathrm{CH}_{3}\right), 1.74-1.85\left(\mathrm{~m}, 2 \mathrm{H}, \mathrm{CH}_{2}\right)$, $3.99\left(\mathrm{t}, 2 \mathrm{H}, \mathrm{J}=6.5, \mathrm{OCH}_{2}\right), 4.42\left(\mathrm{q}, 2 \mathrm{H}, J=7.1, \mathrm{OCH}_{2}\right), 7.21(\mathrm{~s}, 2 \mathrm{H}, \mathrm{Ar}), 7.98(\mathrm{~d}, 2 \mathrm{H}, J=8.8$, Ar), $8.21\left(\mathrm{~d}, 2 \mathrm{H}, J=8.8\right.$, Ar). ${ }^{13} \mathrm{C} \mathrm{NMR}(100 \mathrm{MHz}, \mathrm{CDCl}): 14.11\left(\mathrm{CH}_{3}\right), 14.32\left(\mathrm{CH}_{3}\right), 22.65$ $\left(\mathrm{CH}_{2}\right), 25.89\left(\mathrm{CH}_{2}\right), 28.96\left(\mathrm{CH}_{2}\right), 29.20\left(\mathrm{CH}_{2}\right), 29.25\left(\mathrm{CH}_{2}\right), 31.79\left(\mathrm{CH}_{2}\right), 61.35\left(\mathrm{OCH}_{2}\right), 69.00$ 


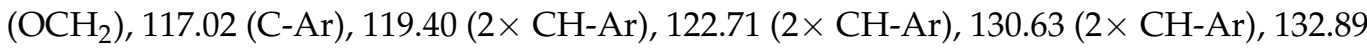
(C-Ar), 142.68 (C-Ar), 154.38 (C-Ar), 159.26 (2× C-Ar), 165.95 (C=O).

Ethyl (E)-4-\{[2,6-dimethyl-4-(octyloxy)phenyl]diazenyl\}benzoate (2d)

According to the general method $\mathrm{A}$, the intermediate $2 \mathrm{~d}$ was prepared and purified to yield $1.21 \mathrm{~g}$ (88\%) of orange solid, m.p. $64-65{ }^{\circ} \mathrm{C} .{ }^{1} \mathrm{H}$ NMR $\left(400 \mathrm{MHz}, \mathrm{CDCl}_{3}\right)$ : 0.89 (t, $\left.3 \mathrm{H}, J=7.1, \mathrm{CH}_{3}\right), 1.23-1.50\left(\mathrm{~m}, 10 \mathrm{H}, 5 \times \mathrm{CH}_{2}\right), 1.43\left(\mathrm{t}, 3 \mathrm{H}, J=7.1, \mathrm{CH}_{3}\right), 1.76-1.84(\mathrm{~m}, 2 \mathrm{H}$, $\left.\mathrm{CH}_{2}\right), 2.53\left(\mathrm{~s}, 6 \mathrm{H}, 2 \times \mathrm{CH}_{3}\right), 4.01\left(\mathrm{t}, 2 \mathrm{H}, J=6.6, \mathrm{OCH}_{2}\right), 4.41\left(\mathrm{q}, 2 \mathrm{H}, J=7.1, \mathrm{OCH}_{2}\right), 6.67$ $(\mathrm{s}, 2 \mathrm{H}, \mathrm{Ar}), 7.86(\mathrm{~d}, 2 \mathrm{H}, J=8.6, \mathrm{Ar}), 8.17(\mathrm{~d}, 2 \mathrm{H}, J=8.6, \mathrm{Ar}) .{ }^{13} \mathrm{C} \mathrm{NMR}\left(100 \mathrm{MHz}, \mathrm{CDCl}_{3}\right)$ : $14.13\left(\mathrm{CH}_{3}\right), 14.35\left(\mathrm{CH}_{3}\right), 20.77\left(2 \times \mathrm{CH}_{3}\right), 22.67\left(\mathrm{CH}_{2}\right), 26.02\left(\mathrm{CH}_{2}\right), 29.22\left(\mathrm{CH}_{2}\right), 29.25$ $\left(\mathrm{CH}_{2}\right), 29.35\left(\mathrm{CH}_{2}\right), 31.82\left(\mathrm{CH}_{2}\right), 61.16\left(\mathrm{OCH}_{2}\right), 68.06\left(\mathrm{OCH}_{2}\right), 115.14(2 \times \mathrm{CH}-\mathrm{Ar}), 121.98$ (2× CH-Ar), 130.53 (2× CH-Ar), 131.22 (C-Ar), 136.09 (C-Ar), 143.98 (C-Ar), 155.74 (C-Ar), $160.03(2 \times \mathrm{C}-\mathrm{Ar}), 166.25(\mathrm{C}=\mathrm{O})$.

\section{(E)-4-\{[2,6-difluoro-4-(octyloxy)phenyl]diazenyl\}benzoic acid (3a)}

According to the general method $\mathrm{B}$, acid $3 \mathrm{a}$ was prepared and purified to yield $80 \mathrm{mg}$ (91\%) of orange solid, m.p. $100-106{ }^{\circ} \mathrm{C} .{ }^{1} \mathrm{H}$ NMR $(400 \mathrm{MHz}, d 6$-DMSO): 0.83 (t, 3H, J = 6.9, $\left.\mathrm{CH}_{3}\right), 1.19-1.43\left(\mathrm{~m}, 10 \mathrm{H}, 5 \times \mathrm{CH}_{2}\right), 1.65-1.75\left(\mathrm{~m}, 2 \mathrm{H}, \mathrm{CH}_{2}\right), 4.08\left(\mathrm{t}, 2 \mathrm{H}, \mathrm{J}=6.6, \mathrm{OCH}_{2}\right), 6.97$ $(\mathrm{d}, 2 \mathrm{H}, J=11.5, \mathrm{Ar}), 7.85(\mathrm{~d}, 2 \mathrm{H}, J=8.6, \mathrm{Ar}), 8.11(\mathrm{~d}, 2 \mathrm{H}, J=8.6, \mathrm{Ar}), 13.21(\mathrm{bs}, 1 \mathrm{H}, \mathrm{COOH})$ ${ }^{13} \mathrm{C}$ NMR (100 MHz, d6-DMSO): $14.41\left(\mathrm{CH}_{3}\right), 22.54\left(\mathrm{CH}_{2}\right), 25.77\left(\mathrm{CH}_{2}\right), 28.73\left(\mathrm{CH}_{2}\right), 29.10$ $\left(\mathrm{CH}_{2}\right), 29.12\left(\mathrm{CH}_{2}\right), 31.69\left(\mathrm{CH}_{2}\right), 69.65\left(\mathrm{OCH}_{2}\right), 100.13\left(\mathrm{dd}, 2 \times \mathrm{CH}-\mathrm{Ar}, J^{\mathrm{C}-\mathrm{F}}=24.0,3.0\right)$, $122.53(2 \times \mathrm{CH}-\mathrm{Ar}), 124.62\left(\mathrm{t}, \mathrm{C}-\mathrm{Ar}, J^{\mathrm{C}-\mathrm{F}}=9.4\right), 131.05(2 \times \mathrm{CH}-\mathrm{Ar}), 133.94(\mathrm{C}-\mathrm{Ar}), 155.40$ $(\mathrm{C}-\mathrm{Ar}), 157.38\left(\mathrm{dd}, 2 \times \mathrm{C}-\mathrm{Ar}, J^{\mathrm{C}-\mathrm{F}}=258.9,7.4\right), 162.58\left(\mathrm{t}, \mathrm{C}-\mathrm{Ar}, J^{\mathrm{C}-\mathrm{F}}=14.7\right), 167.28(\mathrm{C}=\mathrm{O})$.

\section{(E)-4-\{[2,6-dichloro-4-(octyloxy)phenyl]diazenyl\}benzoic acid (3b)}

According to the general method $\mathrm{B}$, acid $3 \mathrm{~b}$ was prepared and purified to yield 0.84 (79\%) of orange solid, m.p. $157-160{ }^{\circ} \mathrm{C} .{ }^{1} \mathrm{H}$ NMR (400 MHz, d6-DMSO): $0.84(\mathrm{t}, 3 \mathrm{H}, J=6.7$, $\left.\mathrm{CH}_{3}\right), 1.21-1.45\left(\mathrm{~m}, 10 \mathrm{H}, 5 \times \mathrm{CH}_{2}\right), 1.66-1.77\left(\mathrm{~m}, 2 \mathrm{H}, \mathrm{CH}_{2}\right), 4.09\left(\mathrm{t}, 2 \mathrm{H}, J=6.5, \mathrm{OCH}_{2}\right), 7.27$ $(\mathrm{s}, 2 \mathrm{H}, \mathrm{Ar}), 7.92(\mathrm{~d}, 2 \mathrm{H}, J=8.5, \mathrm{Ar}), 8.15(\mathrm{~d}, 2 \mathrm{H}, J=8.5, \mathrm{Ar}), 13.34(\mathrm{bs}, 1 \mathrm{H}, \mathrm{COOH}) .{ }^{13} \mathrm{C}$ NMR (100 MHz, d6-DMSO): $14.43\left(\mathrm{CH}_{3}\right), 22.55\left(\mathrm{CH}_{2}\right), 25.78\left(\mathrm{CH}_{2}\right), 28.80\left(\mathrm{CH}_{2}\right), 29.09\left(\mathrm{CH}_{2}\right)$, $29.11\left(\mathrm{CH}_{2}\right), 31.69\left(\mathrm{CH}_{2}\right), 69.34\left(\mathrm{OCH}_{2}\right), 116.34(2 \times \mathrm{CH}-\mathrm{Ar}), 122.92(2 \times \mathrm{CH}-\mathrm{Ar}), 128.46$ (C-Ar), 131.18 (2× CH-Ar), 140.45 (C-Ar), 154.44 (C-Ar), 159.52 (2× C-Ar), 167.07 (C=O).

\section{(E)-4-\{[2,6-dibromo-4-(octyloxy)phenyl]diazenyl\}benzoic acid (3c)}

According to the general method $\mathrm{B}$, acid $3 \mathrm{c}$ was prepared and purified to yield $0.75 \mathrm{mg}$ (79\%) of orange solid, m.p. $165-167{ }^{\circ} \mathrm{C} .{ }^{1} \mathrm{H}$ NMR (400 MHz, d6-DMSO): 0.85 $\left(\mathrm{t}, 3 \mathrm{H}, J=6.9, \mathrm{CH}_{3}\right), 1.17-1.46\left(\mathrm{~m}, 10 \mathrm{H}, 5 \times \mathrm{CH}_{2}\right), 1.65-1.74\left(\mathrm{~m}, 2 \mathrm{H}, \mathrm{CH}_{2}\right), 4.07(\mathrm{t}, 2 \mathrm{H}$, $\left.J=6.5, \mathrm{OCH}_{2}\right), 7.43(\mathrm{~s}, 2 \mathrm{H}, \mathrm{Ar}), 7.92(\mathrm{~d}, 2 \mathrm{H}, J=8.7, \mathrm{Ar}), 8.16(\mathrm{~d}, 2 \mathrm{H}, J=8.7, \mathrm{Ar}), 13.36$ (bs, 1H, COOH). ${ }^{13} \mathrm{C}$ NMR (100 MHz, d6-DMSO): $14.43\left(\mathrm{CH}_{3}\right), 22.55\left(\mathrm{CH}_{2}\right), 25.77\left(\mathrm{CH}_{2}\right)$, $28.83\left(\mathrm{CH}_{2}\right), 29.09\left(\mathrm{CH}_{2}\right), 29.11\left(\mathrm{CH}_{2}\right), 31.68\left(\mathrm{CH}_{2}\right), 69.29\left(\mathrm{OCH}_{2}\right), 116.61(\mathrm{C}-\mathrm{Ar}), 119.73$ ( $2 \times \mathrm{CH}-\mathrm{Ar}), 122.94$ ( $2 \times \mathrm{CH}-\mathrm{Ar}), 131.19$ (2× CH-Ar), 134.45 (C-Ar), 142.61 (C-Ar), 154.00 (C-Ar), 159.61 (2× C-Ar), $167.08(\mathrm{C}=\mathrm{O})$.

\section{(E)-4-\{[2,6-dimethyl-4-(octyloxy)phenyl]diazenyl\}benzoic acid (3d)}

According to the general method $\mathrm{B}$, acid $3 \mathrm{~d}$ was prepared and purified to yield $1.04 \mathrm{~g}$ (94\%) of orange solid, m.p. $160-163{ }^{\circ} \mathrm{C} .{ }^{1} \mathrm{H}$ NMR (400 MHz, d6-DMSO): $0.80-0.88(\mathrm{~m}, 3 \mathrm{H}$, $\left.\mathrm{CH}_{3}\right), 1.16-1.46\left(\mathrm{~m}, 10 \mathrm{H}, 5 \times \mathrm{CH}_{2}\right), 1.65-1.76\left(\mathrm{~m}, 2 \mathrm{H}, \mathrm{CH}_{2}\right), 2.46\left(\mathrm{~s}, 6 \mathrm{H}, 2 \times \mathrm{CH}_{3}\right) 4.01(\mathrm{t}, 2 \mathrm{H}$, $\left.J=6.5, \mathrm{OCH}_{2}\right), 6.78(\mathrm{~s}, 2 \mathrm{H}, \mathrm{Ar}), 7.84(\mathrm{~d}, 2 \mathrm{H}, J=8.5, \mathrm{Ar}), 8.09(\mathrm{~d}, 2 \mathrm{H}, J=8.5, \mathrm{Ar}), 13.13$ (bs, $1 \mathrm{H}, \mathrm{COOH}) .{ }^{13} \mathrm{C}$ NMR (100 MHz, d6-DMSO): $14.42\left(\mathrm{CH}_{3}\right), 20.81\left(2 \times \mathrm{CH}_{3}\right), 22.55\left(\mathrm{CH}_{2}\right)$, $25.92\left(\mathrm{CH}_{2}\right), 29.07\left(\mathrm{CH}_{2}\right), 29.14\left(\mathrm{CH}_{2}\right), 29.17\left(\mathrm{CH}_{2}\right), 31.70\left(\mathrm{CH}_{2}\right), 68.08\left(\mathrm{OCH}_{2}\right), 115.60$ ( $2 \times \mathrm{CH}-\mathrm{Ar}), 122.31$ ( $2 \times \mathrm{CH}-\mathrm{Ar}), 131.03$ (2× CH-Ar), 132.31 (C-Ar), 136.12 (C-Ar), 143.63 (C-Ar), 155.57 (C-Ar), 160.22 (2× C-Ar), 167.29 (C=O). 


\section{4-\{[4-(octyloxy)benzoyl]oxy\}phenyl 3-benzyloxy-4-fluorobenzoate (6)}

To a stirred suspension of 3-benzyloxy-4-fluorobenzoic acid 4 (0.62 g; $2.52 \mathrm{mmol})$, 4-hydroxyfenyl-4-octyloxybenzoate $(0.82 \mathrm{~g} ; 2.40 \mathrm{mmol})$ and DCC $(0.55 \mathrm{~g} ; 2.52 \mathrm{mmol})$ in dry dichloromethane $(50 \mathrm{~mL})$ and, in an inert atmosphere, a catalytic amount of DMAP was added, and the reaction mixture was stirred at room temperature for $24 \mathrm{~h}$. Precipitated $N, N^{\prime}$-dicyclohexylurea was filtered out, and the filtrate was concentrated under reduced pressure. The crude product was purified by multiple crystallization from the mixture of EtOH and EtOAC (1:1) to yield $0.85 \mathrm{~g}(62 \%)$ of white solid, m.p. $126-129^{\circ} \mathrm{C}$. Analytical data are the same as reported [2].

\section{4-\{[4-(octyloxy)benzoyl]oxy\}phenyl 4-fluoro-3-hydroxybenzoate (7)}

To a stirred solution of benzyl derivative $6(0.83 \mathrm{~g} ; 1.45 \mathrm{mmol})$ and ammonium formate $(0.28 \mathrm{~g} ; 4.36 \mathrm{mmol})$ in iso-butyl(methyl)ketone $(50 \mathrm{~mL})$ and inert atmosphere, Pd/C $(10 \%$ $\mathrm{w} / \mathrm{w}$ ) was added. The reaction was refluxed for $2 \mathrm{~h}$, then the catalysator was filtered out, and the filtrate was concentrated under reduced pressure. The crude product was purified by column chromatography (eluent toluene:THF, 10:1) to yield $0.59 \mathrm{~g}(85 \%)$ of white solid, m.p. $118-120^{\circ} \mathrm{C}$. Analytical data are the same as reported [2].

\section{4-((4-(octyloxy)benzoyl)oxy)phenyl (E)-3-((4-((2,6-difluoro-4-(octyloxy)phenyl)diazenyl) benzoyl)oxy)-4-fluorobenzoate (Ia)}

According to the general method $\mathrm{C}$, final material Ia was prepared and purified to yield $180 \mathrm{~g}(90 \%)$ of orange solid. ${ }^{1} \mathrm{H}$ NMR $\left(400 \mathrm{MHz}, \mathrm{CDCl}_{3}\right): \delta 0.85-0.94(\mathrm{~m}, 6 \mathrm{H}$, $\left.2 \times \mathrm{CH}_{3}\right), 1.24-1.41\left(\mathrm{~m}, 16 \mathrm{H}, 8 \times \mathrm{CH}_{2}\right), 1.42-1.52\left(\mathrm{~m}, 4 \mathrm{H}, 2 \times \mathrm{CH}_{2}\right), 1.75-1.88(\mathrm{~m}, 4 \mathrm{H}$, $\left.2 \times \mathrm{CH}_{2}\right), 3.98-4.09\left(\mathrm{~m}, 4 \mathrm{H}, 2 \times \mathrm{OCH}_{2}\right), 6.60\left(\mathrm{~d}, 2 \mathrm{H}, J^{\mathrm{H}-\mathrm{F}}=11.5, \mathrm{Ar}\right), 6.98(\mathrm{~d}, 2 \mathrm{H}, J=8.5, \mathrm{Ar})$, $7.27(\mathrm{~s}, 4 \mathrm{H}, \mathrm{Ar}), 7.34-7.41(\mathrm{~m}, 1 \mathrm{H}, \mathrm{Ar}), 8.00(\mathrm{~d}, 2 \mathrm{H}, J=8.4, \mathrm{Ar}), 8.11-8.21(\mathrm{~m}, 4 \mathrm{H}, \mathrm{Ar}), 8.36$ $(\mathrm{d}, 2 \mathrm{H}, J=8.6) .{ }^{13} \mathrm{C} \mathrm{NMR}\left(100 \mathrm{MHz}, \mathrm{CDCl}_{3}\right): 14.12\left(2 \times \mathrm{CH}_{3}\right), 20.95\left(2 \times \mathrm{CH}_{3}{ }^{\mathrm{Ar}}\right), 22.66$ $\left(\mathrm{CH}_{2}\right), 22.68\left(\mathrm{CH}_{2}\right), 25.99\left(\mathrm{CH}_{2}\right), 26.02\left(\mathrm{CH}_{2}\right), 29.09\left(\mathrm{CH}_{2}\right), 29.22\left(\mathrm{CH}_{2}\right), 29.23\left(\mathrm{CH}_{2}\right), 29.25$ $\left(\mathrm{CH}_{2}\right), 29.33\left(\mathrm{CH}_{2}\right), 29.35\left(\mathrm{CH}_{2}\right), 31.81\left(\mathrm{CH}_{2}\right), 31.82\left(\mathrm{CH}_{2}\right), 68.09\left(\mathrm{OCH}_{2}\right), 68.34\left(\mathrm{OCH}_{2}\right)$, $99.36\left(\mathrm{dd}, 2 \times \mathrm{CH}-\mathrm{Ar}, J^{\mathrm{C}-\mathrm{F}}=23.9,3.0\right) 114.32(2 \times \mathrm{CH}-\mathrm{Ar}), 117.21\left(\mathrm{~d}, \mathrm{CH}-\mathrm{Ar}, J^{\mathrm{C}-\mathrm{F}}=19.5\right)$, 121.26 (C-Ar), 122.48 ( $2 \times \mathrm{CH}-\mathrm{Ar}), 122.66$ ( $2 \times \mathrm{CH}-\mathrm{Ar}), 122.84(2 \times \mathrm{CH}-\mathrm{Ar}), 125.18$ (t, C-Ar, $\left.J^{C-F}=9.2\right), 126.37\left(\mathrm{~d}, \mathrm{C}-\mathrm{Ar}, J^{\mathrm{C}-\mathrm{F}}=3.4\right), 126.44\left(\mathrm{~d}, \mathrm{CH}-\mathrm{Ar}, J^{\mathrm{C}-F}=2.3\right), 129.64(\mathrm{C}-\mathrm{Ar}), 129.84(\mathrm{~d}$, $\left.\mathrm{CH}-\mathrm{Ar}, J^{\mathrm{C}-\mathrm{F}}=8.6\right) 131.53(2 \times \mathrm{CH}-\mathrm{Ar}), 132.32(2 \times \mathrm{CH}-\mathrm{Ar}), 136.56(\mathrm{C}-\mathrm{Ar}), 138.44(\mathrm{~d}, \mathrm{C}-\mathrm{Ar}$, $\left.J^{C-F}=13.5\right), 147.97(\mathrm{C}-\mathrm{Ar}), 148.71(\mathrm{C}-\mathrm{Ar}), 156.71(\mathrm{C}-\mathrm{Ar}), 157.81\left(\mathrm{dd}, 2 \times \mathrm{C}-\mathrm{Ar}, J^{\mathrm{C}-\mathrm{F}}=261.5\right.$, 7.2), $157.93\left(\mathrm{~d}, \mathrm{C}-\mathrm{Ar}, J^{\mathrm{C}-\mathrm{F}}=246.3\right), 162.34\left(\mathrm{t}, \mathrm{C}-\mathrm{Ar}, J^{\mathrm{C}-\mathrm{F}}=14.2\right), 163.43(\mathrm{C}=\mathrm{O}), 163.62(\mathrm{C}=\mathrm{O})$, $164.82(\mathrm{C}=\mathrm{O})$.

\section{4-((4-(octyloxy)benzoyl)oxy)phenyl (E)-3-((4-((2,6-dichloro-4-(octyloxy)phenyl)diazenyl) benzoyl)oxy)-4-fluorobenzoate (Ib)}

According to the general method $\mathrm{C}$, final material $\mathrm{Ib}$ was prepared and purified to yield $60 \mathrm{mg}(37 \%)$ of orange solid. ${ }^{1} \mathrm{H}$ NMR $\left(400 \mathrm{MHz}, \mathrm{CDCl}_{3}\right): \delta 0.83-0.93(\mathrm{~m}, 6 \mathrm{H}$, $\left.2 \times \mathrm{CH}_{3}\right), 1.23-1.54\left(\mathrm{~m}, 20 \mathrm{H}, 10 \times \mathrm{CH}_{2}\right), 1.77-1.86\left(\mathrm{~m}, 4 \mathrm{H}, 2 \times \mathrm{CH}_{2}\right), 3.98-4.09(\mathrm{~m}, 4 \mathrm{H}$, $\left.2 \times \mathrm{OCH}_{2}\right), 6.95-7.01(\mathrm{~m}, 4 \mathrm{H}, \mathrm{Ar}), 7.27(\mathrm{~s}, 4 \mathrm{H}, \mathrm{Ar}), 7.34-7.41(\mathrm{~m}, 1 \mathrm{H}, J=9.0, \mathrm{Ar}), 8.05(\mathrm{~d}, 2 \mathrm{H}$, $J=8.2, \mathrm{Ar}), 8.12-8.21(\mathrm{~m}, 4 \mathrm{H}, \mathrm{Ar}), 8.40(\mathrm{~d}, 2 \mathrm{H}, J=8.3, \mathrm{Ar}) .{ }^{13} \mathrm{C} \mathrm{NMR}\left(100 \mathrm{MHz}, \mathrm{CDCl}_{3}\right)$ : $14.12\left(2 \times \mathrm{CH}_{3}\right), 22.66\left(2 \times \mathrm{CH}_{2}\right), 25.89\left(\mathrm{CH}_{2}\right), 25.99\left(\mathrm{CH}_{2}\right), 28.94\left(\mathrm{CH}_{2}\right), 29.09\left(\mathrm{CH}_{2}\right), 29.21$ $\left(\mathrm{CH}_{2}\right), 29.23\left(\mathrm{CH}_{2}\right), 29.26\left(\mathrm{CH}_{2}\right), 29.33\left(\mathrm{CH}_{2}\right), 31.79\left(\mathrm{CH}_{2}\right), 31.81\left(\mathrm{CH}_{2}\right), 68.35\left(\mathrm{OCH}_{2}\right)$, $69.01\left(\mathrm{OCH}_{2}\right), 114.33(2 \times \mathrm{CH}-\mathrm{Ar}), 115.76(2 \times \mathrm{CH}-\mathrm{Ar}) 117.19\left(\mathrm{~d}, \mathrm{CH}-\mathrm{Ar}, J^{\mathrm{C}-\mathrm{F}}=18.8\right), 121.25$ (C-Ar), 122.48 ( $2 \times \mathrm{CH}-\mathrm{Ar}), 122.86$ ( $2 \times \mathrm{CH}-\mathrm{Ar}), 122.97$ (2× CH-Ar), 126.38 (C-Ar), $126.44(\mathrm{~d}$, $\left.\mathrm{CH}-\mathrm{Ar}, J^{\mathrm{C}-\mathrm{F}}=3.4\right), 129.49(\mathrm{C}-\mathrm{Ar}), 129.93\left(\mathrm{~d}, \mathrm{CH}-\mathrm{Ar}, J^{\mathrm{C}-\mathrm{F}}=8.5\right), 131.60(2 \times \mathrm{CH}-\mathrm{Ar}), 132.33$ $\left(2 \times\right.$ CH-Ar), $138.41\left(\mathrm{~d}, \mathrm{C}-\mathrm{Ar}, J^{\mathrm{C}-\mathrm{F}}=13.4\right), 140.68(\mathrm{C}-\mathrm{Ar}), 147.96(\mathrm{C}-\mathrm{Ar}), 148.72(\mathrm{C}-\mathrm{Ar}), 155.63$ $(\mathrm{C}-\mathrm{Ar}), 157.84\left(\mathrm{~d}, \mathrm{C}-\mathrm{Ar}, J^{\mathrm{C}-\mathrm{F}}=258.5\right), 159.30(2 \times \mathrm{C}-\mathrm{Ar}), 163.34(\mathrm{C}=\mathrm{O}), 163.42(\mathrm{C}=\mathrm{O}), 163.63$ (C-Ar), $164.83(\mathrm{C}=\mathrm{O})$. 
4-((4-(octyloxy)benzoyl)oxy)phenyl (E)-3-((4-((2,6-dibromo-4-(octyloxy)phenyl)diazenyl) benzoyl)oxy)-4-fluorobenzoate (Ic)

According to the general method $C$, final material Ic was prepared and purified to yield $350 \mathrm{mg}(73 \%)$ of orange solid. ${ }^{1} \mathrm{H} \mathrm{NMR}\left(400 \mathrm{MHz}, \mathrm{CDCl}_{3}\right): \delta 0.86-0.95(\mathrm{~m}, 6 \mathrm{H}$, $\left.2 \times \mathrm{CH}_{3}\right), 1.22-1.42\left(\mathrm{~m}, 16 \mathrm{H}, 8 \times \mathrm{CH}_{2}\right), 1.43-1.52\left(\mathrm{~m}, 4 \mathrm{H}, 2 \times \mathrm{CH}_{2}\right), 1.76-1.89(\mathrm{~m}, 4 \mathrm{H}$, $\left.2 \times \mathrm{CH}_{2}\right), 3.98-4.08\left(\mathrm{~m}, 4 \mathrm{H}, 2 \times \mathrm{OCH}_{2}\right), 6.60(\mathrm{~m}, 2 \mathrm{H}, \mathrm{Ar}), 6.98(\mathrm{~d}, 2 \mathrm{H}, J=8.3, \mathrm{Ar}), 6.99-7.25$ (m, 2H, Ar), 7.27 (s, 4H, Ar), 7.38 (m, 1H, Ar), 8.04-8.09 (m, 2H, Ar), 8.11-8.21 (m, 4H, Ar), 8.38-8.42 (m, 2H, Ar). ${ }^{13} \mathrm{C} \mathrm{NMR}\left(100 \mathrm{MHz}, \mathrm{CDCl}_{3}\right): 14.12\left(2 \times \mathrm{CH}_{3}\right), 22.66\left(2 \times \mathrm{CH}_{2}\right), 25.89$ $\left(\mathrm{CH}_{2}\right), 25.99\left(\mathrm{CH}_{2}\right), 28.95\left(\mathrm{CH}_{2}\right), 29.09\left(\mathrm{CH}_{2}\right), 29.21\left(\mathrm{CH}_{2}\right), 29.23\left(\mathrm{CH}_{2}\right), 29.26\left(\mathrm{CH}_{2}\right), 29.33$ $\left(\mathrm{CH}_{2}\right), 31.79\left(\mathrm{CH}_{2}\right), 31.81\left(\mathrm{CH}_{2}\right), 68.34\left(\mathrm{OCH}_{2}\right), 69.01\left(\mathrm{~m}, \mathrm{OCH}_{2}\right), 114.32(2 \times \mathrm{CH}-\mathrm{Ar}), 116.12$ (d, CH-Ar, $J=73.0), 117.21\left(\mathrm{~d}, \mathrm{CH}-\mathrm{Ar}, J^{C-F}=19.5\right), 118.16(\mathrm{~d}, \mathrm{C}-\mathrm{Ar}, J=174.4), 119.12(\mathrm{~d}$, C-Ar, $J=72.9) 121.26$ (C-Ar), 122.96 (2× CH-Ar), 123.01 (2× CH-Ar), 123.04 (m, 2× CH-Ar), $126.37\left(\mathrm{~d}, \mathrm{C}-\mathrm{Ar}, J^{C-F}=1,8\right), 126.40\left(\mathrm{~d}, \mathrm{CH}-\mathrm{Ar}, J^{C-F}=3.4\right), 128.77(\mathrm{~d}, \mathrm{C}-\mathrm{Ar}, J=146.0), 129.93(\mathrm{~d}$, $\left.\mathrm{CH}-\mathrm{Ar}, J^{\mathrm{C}-\mathrm{F}}=8.4\right), 130.37(\mathrm{~m}, \mathrm{C}-\mathrm{Ar}), 131.61(2 \times \mathrm{CH}-\mathrm{Ar}), 132.32(2 \times \mathrm{CH}-\mathrm{Ar}), 138.41(\mathrm{~d}, \mathrm{C}-\mathrm{Ar}$, $\left.J^{C-F}=13.4\right), 141.59(\mathrm{~m}, \mathrm{C}-\mathrm{Ar}), 147.96(\mathrm{C}-\mathrm{Ar}), 148.72(\mathrm{C}-\mathrm{Ar}), 155.39$ (m, C-Ar), 157.83 (d, C-Ar, $\left.J^{C-F}=258.7\right), 159.39(\mathrm{~m}, 2 \times C-A r), 163.34(\mathrm{~d}, J=8.4, C=\mathrm{O}), 163.63(\mathrm{C}=\mathrm{O}), 164.81(\mathrm{C}=\mathrm{O})$.

4-((4-(octyloxy)benzoyl)oxy)phenyl (E)-3-((4-((2,6-dimethyl-4-(octyloxy)phenyl)diazenyl) benzoyl)oxy)-4-fluorobenzoate (Id)

According to the general method $\mathrm{C}$, final material Id was prepared and purified to yield $220 \mathrm{mg}(87 \%)$ of orange solid. ${ }^{1} \mathrm{H}$ NMR (400 MHz, $\left.\mathrm{CDCl}_{3}\right)$ : $\delta$ 0.85-0.94 (m, $\left.6 \mathrm{H}, 2 \times \mathrm{CH}_{3}\right), 1.25-1.41\left(\mathrm{~m}, 16 \mathrm{H}, 8 \times \mathrm{CH}_{2}\right), 1.44-1.52\left(\mathrm{~m}, 4 \mathrm{H}, 2 \times \mathrm{CH}_{2}\right), 1.76-1.89(\mathrm{~m}, 4 \mathrm{H}$, $\left.2 \times \mathrm{CH}_{2}\right), 2.57\left(\mathrm{~s}, 6 \mathrm{H}, 2 \times \mathrm{CH}_{3}\right), 3.98-4.09\left(\mathrm{~m}, 4 \mathrm{H}, 2 \times \mathrm{OCH}_{2}\right), 6.69(\mathrm{~s}, 2 \mathrm{H}, \mathrm{Ar}), 6.98(\mathrm{~d}, 2 \mathrm{H}$, $J=9.0, \mathrm{Ar}), 7.27(\mathrm{~s}, 4 \mathrm{H}, \mathrm{Ar}), 7.34-7.41(\mathrm{~m}, 1 \mathrm{H}, \mathrm{Ar}), 7.95(\mathrm{~d}, 2 \mathrm{H}, J=8.7, \mathrm{Ar}), 8.10-8.21(\mathrm{~m}, 4 \mathrm{H}$, $\mathrm{Ar}), 8.35(\mathrm{~d}, 2 \mathrm{H}, \mathrm{J}=8.7) .{ }^{13} \mathrm{C} \mathrm{NMR}\left(100 \mathrm{MHz}, \mathrm{CDCl}_{3}\right): 14.12\left(2 \times \mathrm{CH}_{3}\right), 20.95\left(2 \times \mathrm{CH}_{3}{ }^{\mathrm{Ar}}\right)$, 22.66 $\left(\mathrm{CH}_{2}\right), 22.68\left(\mathrm{CH}_{2}\right), 25.99\left(\mathrm{CH}_{2}\right), 26.02\left(\mathrm{CH}_{2}\right), 29.09\left(\mathrm{CH}_{2}\right), 29.22\left(\mathrm{CH}_{2}\right), 29.23\left(\mathrm{CH}_{2}\right)$, $29.25\left(\mathrm{CH}_{2}\right), 29.33\left(\mathrm{CH}_{2}\right), 29.35\left(\mathrm{CH}_{2}\right), 31.81\left(\mathrm{CH}_{2}\right), 31.82\left(\mathrm{CH}_{2}\right), 68.09\left(\mathrm{OCH}_{2}\right), 68.34\left(\mathrm{OCH}_{2}\right)$, 114.32 (2× CH-Ar), 115.23 (2× CH-Ar), $117.19\left(\mathrm{~d}, \mathrm{CH}-\mathrm{Ar}, J^{\mathrm{C}-\mathrm{F}}=19.6\right), 121.27$ (C-Ar), 122.33

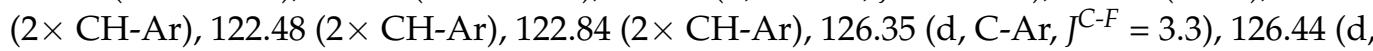
$\left.\mathrm{CH}-\mathrm{Ar}, J^{\mathrm{C}-\mathrm{F}}=2.1\right), 128.69(\mathrm{C}-\mathrm{Ar}), 129.82\left(\mathrm{~d}, \mathrm{CH}-\mathrm{Ar}, J^{C-F}=8.5\right) 131.55(2 \times \mathrm{CH}-\mathrm{Ar}), 132.32$ (2× CH-Ar), $136.56(2 \times \mathrm{C}-\mathrm{Ar}), 138.51\left(\mathrm{~d}, \mathrm{C}-\mathrm{Ar}, J^{C-F}=13.4\right), 143.90(\mathrm{C}-\mathrm{Ar}), 147.98(\mathrm{C}-\mathrm{Ar})$, $148.71(\mathrm{C}-\mathrm{Ar}), 156.62(\mathrm{C}-\mathrm{Ar}), 157.91\left(\mathrm{~d}, \mathrm{C}-\mathrm{Ar}, J^{\mathrm{C}-\mathrm{F}}=259.1\right), 160.34(2 \times \mathrm{C}-\mathrm{Ar}), 163.44(\mathrm{C}=\mathrm{O})$, $163.60(\mathrm{C}=\mathrm{O}), 163.63$ (C-Ar), $164.81(\mathrm{C}=\mathrm{O})$.

\subsection{Characterization of Mesomorphic and Photochemical Behavior}

The sequence of phases and phase transition temperatures of materials Ia-d were determined by observations of characteristic textures and their changes in a polarizing optical microscope (POM) NIKON Elipse E600Pol (Nikon, Tokyo, Japan) and checked by differential scanning calorimetry (DSC) and a Perkin-Elmer 8000 (Perkin-Elmer, Waltham, MA, USA) calorimeter was utilized on the cooling and heating runs at a rate of $5 \mathrm{~K} \mathrm{~min}^{-1}$. Temperature and enthalpy change values were calibrated on the temperatures and the enthalpy changes of the melting points of water, indium, and zinc.

Absorption spectra were recorded on a UV-Vis spectrometer (Agilent Cary 8454) in matched $1 \mathrm{~cm}$ quartz cells (cells for fluorescence measurements with a screw cap with septa from Hellma) equipped with a stir bar, at ambient temperature $\left(22 \pm 1^{\circ} \mathrm{C}\right)$. A solution of the selected compound (Ia-Id, solvent-chloroform, c $=4-8 \times 10^{-5} \mathrm{M}$, volume-3 $\mathrm{mL}$ ) was irradiated with a light source of the selected wavelength $\left(\lambda_{\max }=368 \pm 10,460 \pm 15\right.$, $550 \pm 20 \mathrm{~nm}$, hereafter denoted as 365, 460 and $560 \mathrm{~nm}$, which are the manufacturer specified values) arranged perpendicularly to the spectrometer beam so that the spectra could be recorded during irradiation. The samples were irradiated for at least $30 \mathrm{~min}$, the photostationary state (PSS) was determined when at least 5 absorption spectra recorded in 5 min intervals were identical.

The photoresponses of the materials Ia-d in mesophase were studied by POM, using a Nikon Eclipse E400Pol equipped with a Nikon U2 (CCD 5Mpx camera), UV-Vis Ocean 
Optics SD2000 miniature fiber optic spectrometer, and a homemade microscopy hot-stage (PID controlled in the range of $20-120^{\circ} \mathrm{C}$ ). The BCLC materials were cooled down to a desired temperature and illuminated with UV/Vis light (wavelength 365, 460 and $560 \mathrm{~nm}$, intensity $11.3 \mathrm{~mW} \mathrm{~cm}^{-2}$ ) using different LED diode sources (Height-Led, Shenzhen, China).

Photostationary states were determined using ${ }^{1} \mathrm{H}$ NMR studies. Solutions of the final materials Ia-d $\left(0.6 \times 10^{-3} \mathrm{~mol} \mathrm{~L}^{-1}\right)$ in $\mathrm{CDCl}_{3}(0.6 \mathrm{~mL})$ were heated to the boiling point in a cuvette and an almost quantitative conversion to the $(E)$ form was observed. Samples were then exposed to irradiation with 365,460 and $560 \mathrm{~nm}$ wavelength in sequence to obtain the PSS. PSS ${ }_{365}$ was reached after $1500 \mathrm{~s}, \mathrm{PSS}_{460}$ after $240 \mathrm{~s}$ and $\mathrm{PSS}_{560}$ after $540 \mathrm{~s}$ (no significant changes with further irradiation were observed). The ${ }^{1} \mathrm{H}$ NMR spectra were acquired on an Agilent 400-MR DDR2 spectrometer (Agilent Technologies, Santa Clara, CA, USA).

\section{Results and Discussion}

\subsection{Mesomorphic Properties}

The mesomorphic properties of the prepared materials were studied using POM and DSC. Results of these measurements are summarized in Table 1. An example of a DSC heating/cooling run for material Id is presented in Figure 3.

Table 1. Phase transition temperatures $\left({ }^{\circ} \mathrm{C}\right)$ measured on cooling $\left(5^{\circ} \mathrm{C} \mathrm{min}^{-1}\right)$ and melting points, m.p. $\left({ }^{\circ} \mathrm{C}\right)$, measured on heating $\left(5^{\circ} \mathrm{C} \mathrm{min}^{-1}\right)$ determined by differential scanning calorimetry (DSC) for the Ia-d materials, $\mathrm{Cr}$ - crystalline phase; Tcr-crystallization temperature, ${ }^{\circ} \mathrm{C} ; \mathrm{M}_{1}-\mathrm{M}_{3}$ - formed mesophases on cooling; $\mathrm{Ttr}$ - transition temperature, ${ }^{\circ} \mathrm{C}$; Iso-isotropic liquid state; $\mathrm{N}$-nematic phase; $\bullet /$ - - phase exists / does not exist. The enthalpy changes, $\Delta \mathrm{H}$, in kJ mol${ }^{-1}$, are in brackets at the corresponding temperature.

\begin{tabular}{|c|c|c|c|c|c|c|c|c|}
\hline Material & $\begin{array}{l}\text { m.p. } \\
\Delta \mathrm{H}\end{array}$ & $\mathrm{Cr}$ & $\begin{array}{l}T_{C r} \\
\Delta \mathbf{H}\end{array}$ & $\mathbf{M}_{2}$ & $\mathbf{T}_{\mathrm{Tr}}$ & $\mathbf{M}_{1}$ & $\begin{array}{l}\mathbf{T}_{\mathrm{Tr}} \\
\Delta \mathbf{H}\end{array}$ & Iso \\
\hline SV775 & 124 & $\bullet$ & 105 & SmX & 110 & $\mathrm{~N}$ & 128 & $\bullet$ \\
\hline Ia & $\begin{array}{c}115 \\
+49.8\end{array}$ & $\bullet$ & $\begin{array}{c}92 \\
-70.6\end{array}$ & - & - & $\mathrm{N}$ & $\begin{array}{c}110 \\
-1.2\end{array}$ & $\bullet$ \\
\hline $\mathrm{Ib}$ & $\begin{array}{c}100 \\
+36.9\end{array}$ & $\bullet$ & $\begin{array}{c}49 \\
-15.6\end{array}$ & - & - & $\mathrm{N}$ & $\begin{array}{c}73 \\
-0.6\end{array}$ & $\bullet$ \\
\hline Ic & $\begin{array}{c}101 \\
+34.1\end{array}$ & $\bullet$ & $\begin{array}{c}59 \\
-10.2\end{array}$ & - & - & $\mathrm{N}$ & $\begin{array}{c}80 \\
-0.58\end{array}$ & • \\
\hline Id & $\begin{array}{c}119 \\
+46.4\end{array}$ & $\bullet$ & $\begin{array}{c}75 \\
-31.4\end{array}$ & - & - & $\mathrm{N}$ & $\begin{array}{c}100 \\
-0.25\end{array}$ & $\bullet$ \\
\hline
\end{tabular}

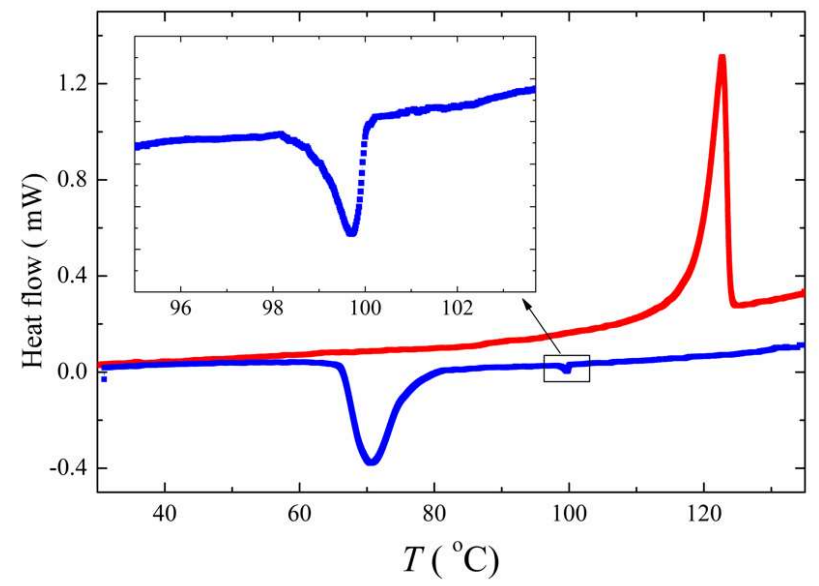

Figure 3. DSC plot of final material Id (heating — red line, cooling—blue line). The enthalpy change corresponding to the phase transition from the isotropic state to the nematic phase is shown in the inset.

In comparison with previously reported SV775, which exhibited one additional smectic mesophase before crystallization [2], the materials prepared here formed a nematic phase 
only. The introduction of lateral substituents to the photosensitive arm decreased the melting point and transition temperatures from Iso to the nematic mesophase on cooling. For the newly prepared compounds Ia-Id, only a monotropic nematic phase was found on cooling from the isotropic phase. This nematic mesophase was rather unstable, and during POM crystallization appeared (Figure 4). It is assumed that the steric demands of the substitution hindered self-organization into more organized system (e.g., smectic layers) and, therefore, cooling from the nematic phase led directly to crystallization.
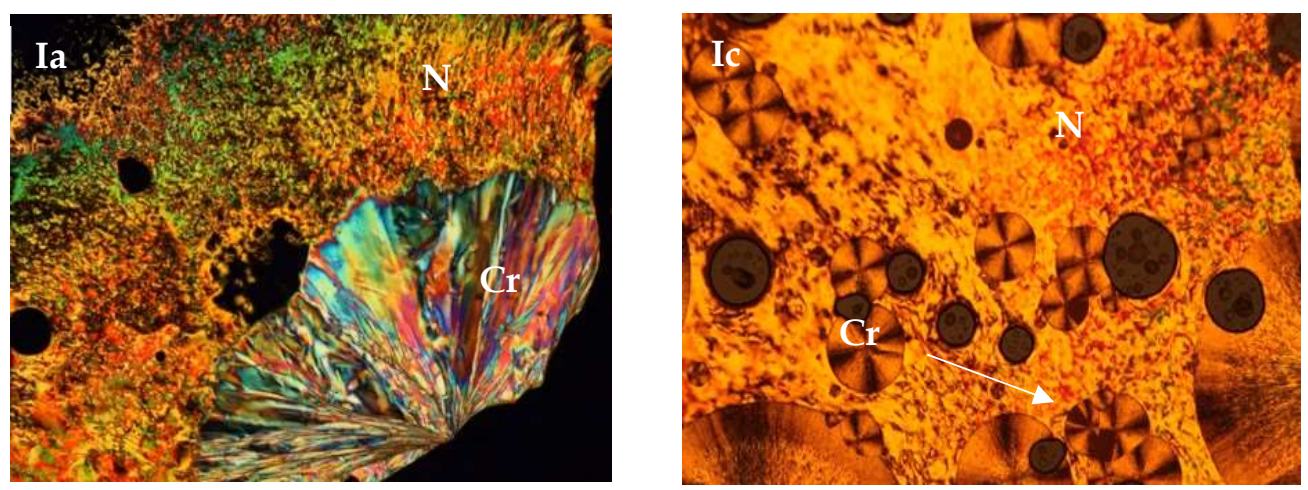

Figure 4. Photos of materials Ia and Ic during the cooling and crystallization processes from the nematic mesophase observed in POM.

\subsection{Photosensitivity of the Materials}

\subsubsection{Photo-Response in Solution}

Since all prepared LC materials comprise photosensitive azo (diazenyl) group, their geometry can be easily changed by UV/Vis light irradiation (Figure 5). (E)-(Z) isomerization from the initial state led to the breaking of the supramolecular order, and thus to the phase transition. The most common is the light-induced isothermal transition from mesophase to the isotropic liquid [36]. In some cases, it is possible to observe a transition to a different mesophase [37-39].<smiles>[Y]c1cc(OCCCC)cc([X])c1N=Nc1ccc(C(=O)Oc2cc(C(=O)Oc3ccc(OC(=O)c4ccc(OCCC)cc4)cc3)ccc2F)cc1</smiles>

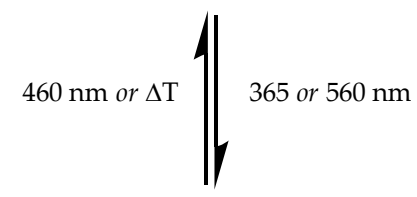<smiles>[X]c1cc(OCCCC)cc([X])c1/N=N\c1ccc(C(=O)Oc2cc(C(=O)Oc3ccc(OC(=O)c4ccc(OCCCC)cc4)cc3)ccc2F)cc1</smiles>

Figure 5. Photochemical isomerization of prepared BCLCs. 
First, the photo-response of the new BCLCs was studied in solution using UV/Vis measurements at various wavelengths (Figure 6). For all materials, a decrease in the intensity of an absorption band characteristic for the $(E)$-isomer was observed under ambient light. Therefore, two visible light wavelengths were used to study this feature closer. It is worth noting that the photo-response strongly depended on lateral substitution in the vicinity of the azo group. For the halogenated compounds Ia-c, high-energy visible light $(460 \mathrm{~nm})$ had a similar effect as ambient light. On the other hand, low-energy visible light $(560 \mathrm{~nm})$ induced the $(E)$-to- $(Z)$ transition, particularly in the cases of materials $\mathrm{Ib}$ and Ic substituted with chlorine and bromine, respectively. This behavior was caused by the shift of the absorption maximum caused by the substituents, which can be observed especially for the (Z)-isomers. For the methyl-substituted compound Id, only a weak effect of visible light was observed.

The $(E)$-to- $(Z)$ transition induced by the UV-light $(365 \mathrm{~nm})$ was the most effective for all compounds. In the case of fluoro-substituted material Ia, the absorption band of the (E)-isomer almost disappeared. In the case of materials Ib and Ic, substituted with chlorines and bromines, respectively, the effectivity of irradiation was lower. The lowest effect of UV light was again observed for the methyl-substituted material Id.
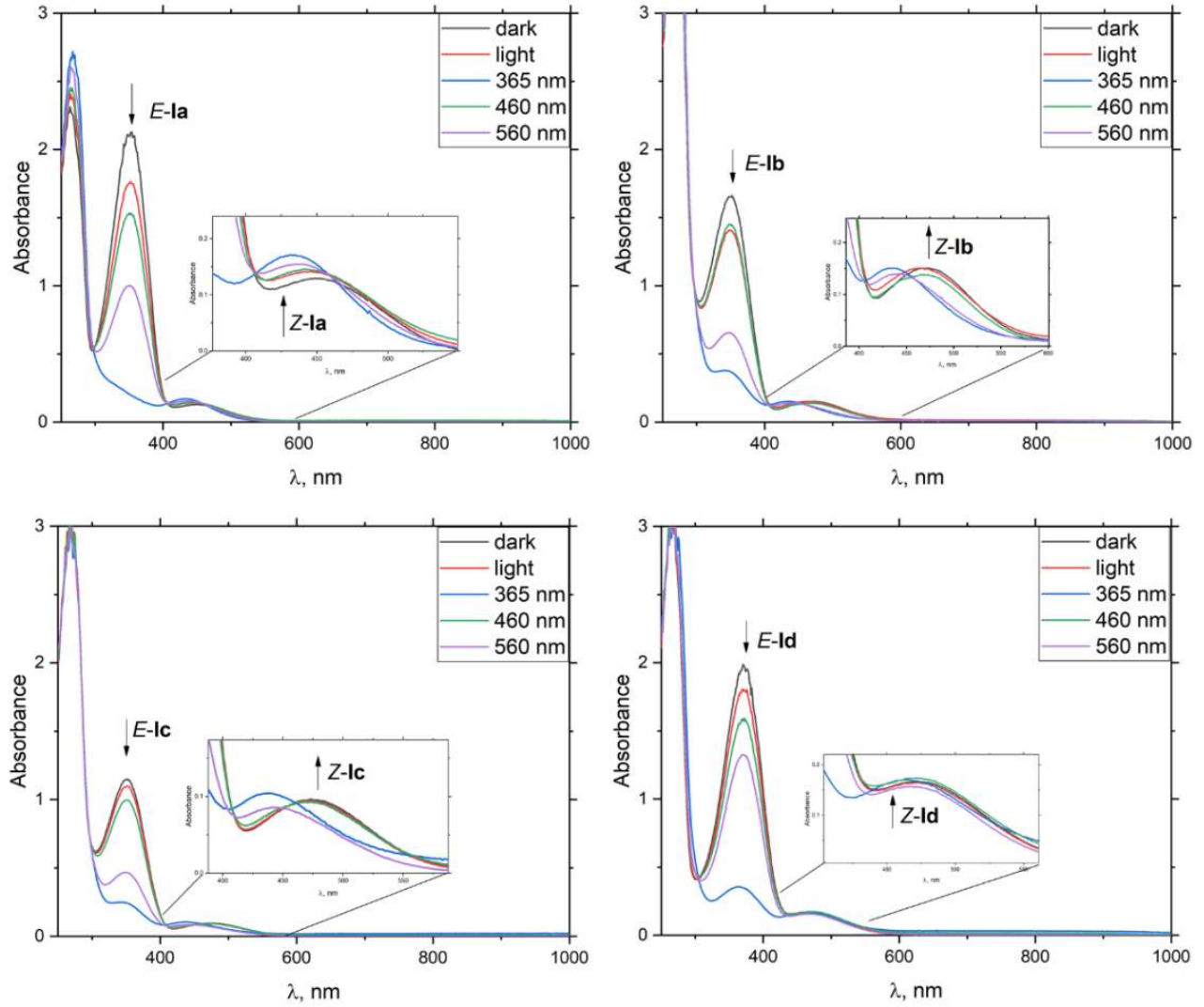

Figure 6. UV/Vis spectra of the studied materials measured in dark (black), at ambient light (red), UV-light (365 nm, blue) and two visible light wavelengths (460 nm, green) and (560 nm, purple).

In order to determine the exact $(E) /(Z)$ ratio after irradiation with various light sources, the PSS of reversible photochemical switching between the $(E)$ and $(Z)$ isomers were established with ${ }^{1} \mathrm{H}$ NMR studies (Table 2). In all cases, UV light $(365 \mathrm{~nm})$ generated the higher ratio of $(Z)$ isomer in comparison with visible green light $(560 \mathrm{~nm})$. The process of inverse isomerization (from $(Z)$ and $(E)$ ) is possible upon irradiation with blue visible light $(460 \mathrm{~nm})$. The best photo-response and switchability was shown in the fluorinated (Ia) and chlorinated $(\mathrm{Ib})$ derivatives, while the larger excesses of $(\mathrm{Z})$ formed in PSS $_{365}$ and PSS $_{560}$. The inverse process of isomerization to the $(E)$ configuration was effective However, 
the bromo- and methyl-substituted compounds Ic and Id, respectively, exhibited higher $(Z) /(E)$ ratios than Ia and Ib.

Table 2. Ratio of $(E)$ and $(Z)$ isomers in various PSS states in deuterochloroform solution at room temperature.

\begin{tabular}{ccccc}
\hline \multicolumn{5}{c}{$(\boldsymbol{E}) /(\mathbf{Z})$ Ratios } \\
\hline $\mathbf{X}$ & PSS $_{\mathbf{3 6 5}}$ & PSS $_{\mathbf{4 6 0}}$ & PSS $_{\mathbf{5 6 0}}$ \\
\hline Ia & $\mathrm{F}$ & $13 / 87$ & $70 / 30$ & $45 / 55$ \\
Ib & $\mathrm{Cl}$ & $24 / 76$ & $68 / 32$ & $36 / 64$ \\
Ic & $\mathrm{Br}$ & $52 / 48$ & $84 / 16$ & $64 / 36$ \\
Id & $\mathrm{CH}_{3}$ & $36 / 64$ & $80 / 20$ & $70 / 30$ \\
\hline
\end{tabular}

The example of the ${ }^{1} \mathrm{H}$ NMR spectra and determination of $(E) /(Z)$ ratio for the material Ia are shown in Figure 7. Signals of aromatic hydrogens neighboring fluorine atoms at 6.60 and $6.36 \mathrm{ppm}$ were well separated for both $(E)$ and $(Z)$ isomer. Intensity changes of signals after irradiation are clearly observable, and indicated photochemical switching. PSSs were established when no significant changes in spectra were observed on further irradiation.

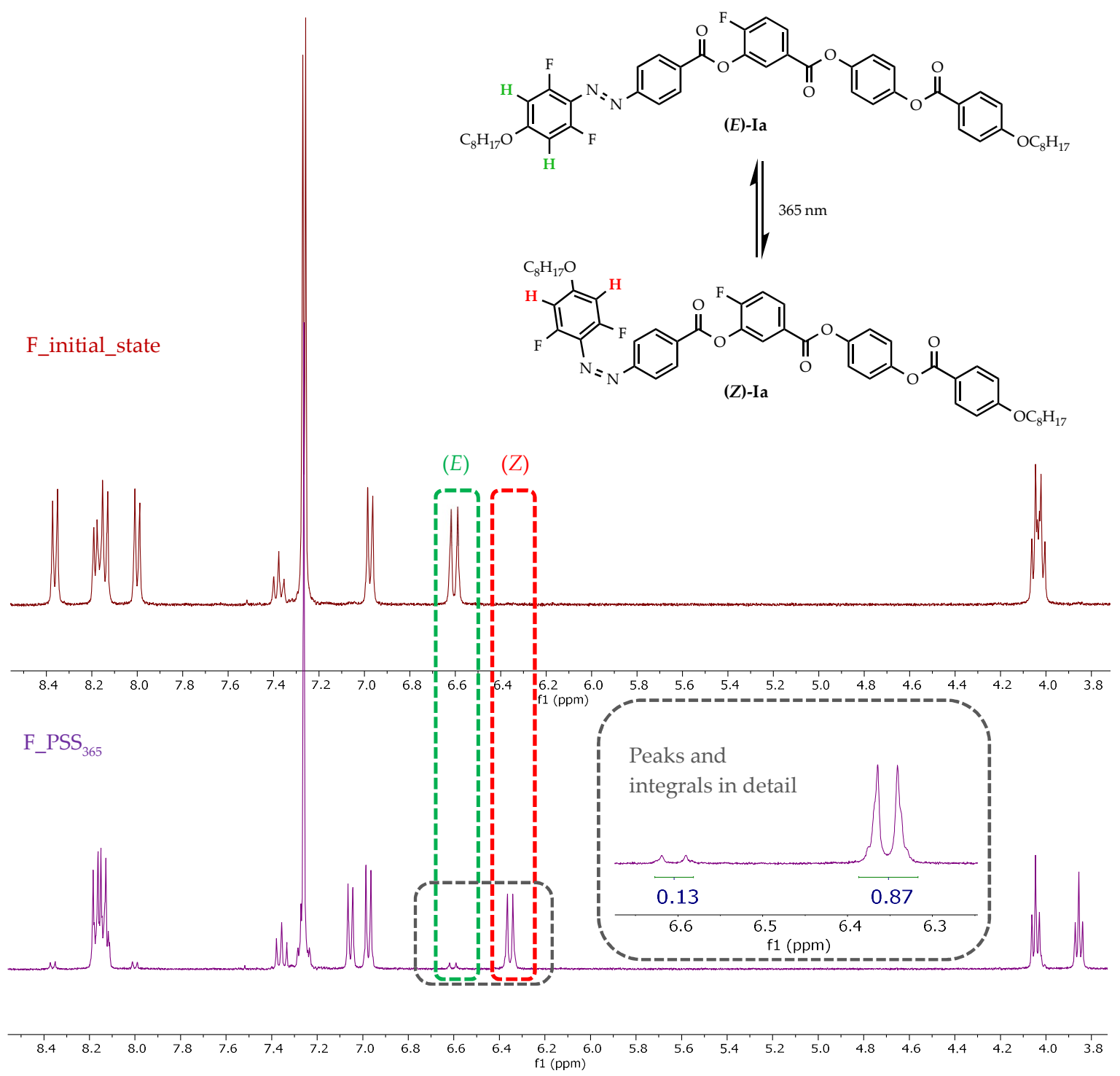

Figure 7. Determination of the $(E) /(Z)$ ratio from ${ }^{1} \mathrm{H}$ NMR spectrum of the material Ia. Integrals of significant signals at 6.60 and $6.36 \mathrm{ppm}$ correspond with percentage of $(E)$ and $(Z)$ isomers in the solution. 


\subsubsection{Photo-Response in Mesophase}

The effect of irradiation was studied also in mesophase, primarily using a UV light $(365 \mathrm{~nm})$. In this case, all newly prepared materials underwent immediate light-induced transition from $\mathrm{N}$ to Iso (Figure 8). Photoisomerization from $(E)$ to $(Z)$ can be managed not only by UV $(365 \mathrm{~nm})$, but also by visible green light $(560 \mathrm{~nm})$. In both cases, the light-induced $(Z)$-isomer, which is responsible for the transition of the system to the isotropic phase, remained stable for several hours and no thermal back relaxation was observed. Such high stability of the (Z)-isomers during constant heating is remarkable, but it corresponds with the published literature $[14,40,41]$. However, the main drawback of the reported substances is their monotropic behavior. Due to their monotropic character, the materials did not undergo complete back-relaxation to the nematic phase. Instead, a slow transition to the crystalline state was observed. We assume that higher stability of the nematic phase, i.e., reaching the enantiotropic nematic phase, could be achieved by elongation of the terminal alkyl chains.

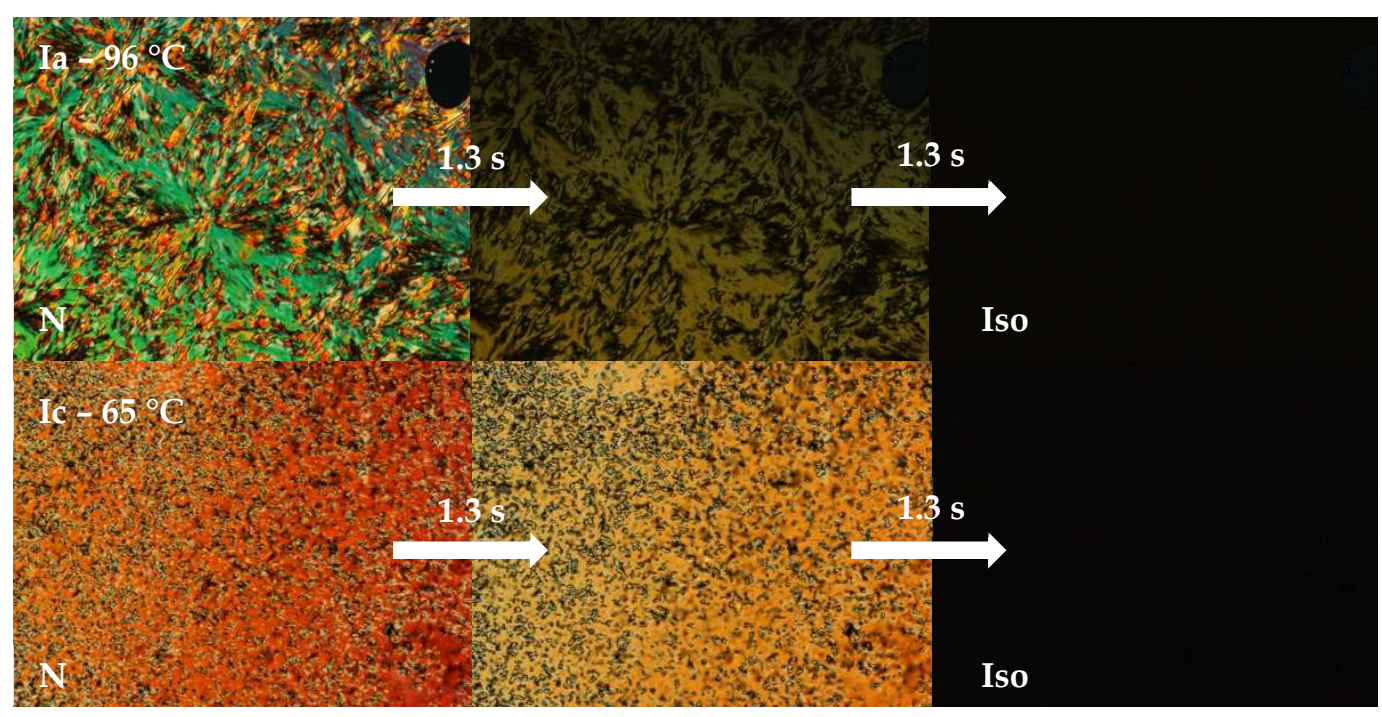

Figure 8. Photos of isothermal photo-triggered phase transition of materials Ia and Ic, observed in POM. Complete transition to isotropic liquid was finished within less than $3 \mathrm{~s}$.

\section{Conclusions}

We report on the synthesis, mesomorphic properties, and photochemical behavior of four novel bent-core liquid crystals. The materials feature a common lateral substituent (fluorine) on the central core, which helps to reduce phase transition temperatures. In addition to that, fluorine was previously identified as a helpful substituent in the formation of a nematic phase, which is particularly interesting for bent-core materials [29]. Further lateral substituents were introduced to the azobenzene wing close to the azo group. These substituents have been shown to influence the spectral properties of the materials, and significantly stabilize photochemically-induced $(Z)$-isomer of the azo group. The experiments performed in the mesophase confirmed that the materials can be isothermally switched from the condensed phase to isotropic liquid. Moreover, the materials expressed high stability (several hours) in the photochemically induced isotropic liquid before transition to the crystalline state.

Author Contributions: Conceptualization, M.K.; synthesis, D.J.; formal analysis, A.P., V.N.; investigation, V.N., M.Š.; writing—original draft preparation, M.Š.; writing—review and editing, M.K.; supervision, J.M., M.K.; funding acquisition, M.K. All authors have read and agreed to the published version of the manuscript.

Funding: This research was funded by the Czech Science Foundation, grant number 19-03564S. 
Data Availability Statement: Experimental data are available upon request.

Conflicts of Interest: The authors declare no conflict of interest.

\section{References}

1. Goodby, J.W.; Collings, P.J.; Kato, T.; Tschierste, C.; Gleeson, H.; Raynes, P.; Vill, V. (Eds.) Handbook of Liquid Crystals; Wiley-VCH Verlag: Weinheim, Germany, 2014.

2. Jing, H.; Xu, M.; Xiang, Y.; Wang, E.; Liu, D.; Poryvai, A.; Kohout, M.; Éber, N.; Buka, Á. Light Tunable Gratings Based on Flexoelectric Effect in Photoresponsive Bent-Core Nematics. Adv. Opt. Mater. 2019, 7, 1801790. [CrossRef]

3. Asquini, R.; d'Alessandro, A. Tunable Photonic Devices Based on Liquid Crystals and Composites; SPIE: San Diego, CA, USA, 2013; Volume 8828.

4. Kawata, S.; Kawata, Y. Three-Dimensional Optical Data Storage Using Photochromic Materials. Chem. Rev. 2000, 100, 1777-1788. [CrossRef] [PubMed]

5. Ikeda, T. Photomodulation of liquid crystal orientations for photonic applications. J. Mater. Chem. 2003, 13, 2037-2057. [CrossRef]

6. Petsch, S.; Rix, R.; Khatri, B.; Schuhladen, S.; Müller, P.; Zentel, R.; Zappe, H. Smart artificial muscle actuators: Liquid crystal elastomers with integrated temperature feedback. Sens. Actuators A Phys. 2015, 231, 44-51. [CrossRef]

7. Zeng, H.; Wani, O.M.; Wasylczyk, P.; Kaczmarek, R.; Priimagi, A. Self-Regulating Iris Based on Light-Actuated Liquid Crystal Elastomer. Adv. Mater. 2017, 29, 1701814. [CrossRef] [PubMed]

8. Alaasar, M. Azobenzene-containing bent-core liquid crystals: An overview. Liq. Cryst. 2016, 43, 2208-2243. [CrossRef]

9. Poryvai, A.; Bubnov, A.; Kohout, M. Chiral Photoresponsive Liquid Crystalline Materials Derived from Cyanoazobenzene Central Core: Effect of UV Light Illumination on Mesomorphic Behavior. Crystals 2020, 10, 1161. [CrossRef]

10. Poryvai, A.; Bubnov, A.; Pociecha, D.; Svoboda, J.; Kohout, M. The effect of the length of terminal n-alkyl carboxylate chain on self-assembling and photosensitive properties of chiral lactic acid derivatives. J. Mol. Liq. 2019, 275, 829-838. [CrossRef]

11. Šmahel, M.; Poryvai, A.; Xiang, Y.; Pociecha, D.; Troha, T.; Novotná, V.; Svoboda, J.; Kohout, M. Photosensitive bent-core nematic liquid crystals with various linking units in the side arms: Structure-properties relationships. J. Mol. Liq. 2020, $306,112743$. [CrossRef]

12. Bandara, H.M.D.; Burdette, S.C. Photoisomerization in different classes of azobenzene. Chem. Soc. Rev. 2012, 41, 1809-1825. [CrossRef]

13. Leistner, A.-L.; Kirchner, S.; Karcher, J.; Bantle, T.; Schulte, M.L.; Gödtel, P.; Fengler, C.; Pianowski, Z.L. Fluorinated Azobenzenes Switchable with Red Light. Chemistry 2021, 27, 8094-8099. [CrossRef]

14. Cigl, M.; Bubnov, A.; Kašpar, M.; Hampl, F.; Hamplová, V.; Pacherová, O.; Svoboda, J. Photosensitive chiral self-assembling materials: Significant effects of small lateral substituents. J. Mater. Chem. C 2016, 4, 5326-5333. [CrossRef]

15. Prasad, V.; Nagendrappa Gowdru, N.; Manjunath, M. Thermally stable azo-substituted bent-core nematogens: Observation of chiral domains in the nematic mesophases composed of smectic nano clusters. Liq. Cryst. 2018, 45, 666-679. [CrossRef]

16. Hrozhyk, U.; Serak, S.; Tabiryan, N.; Bunning, T.J. Wide Temperature Range Azobenzene Nematic and Smectic LC Materials. Mol. Cryst. Liq. Cryst. 2006, 454, 235-245. [CrossRef]

17. Nersesyan, V.; Dadalyan, T.; Beeckman, J.; Beunis, F.; Neyts, K.; Drampyan, R. Refractive Bessel lattice in azobenzene liquid crystal. J. Mod. Opt. 2018, 65, 2034-2043. [CrossRef]

18. Serak, S.V.; Tabiryan, N.V.; Assanto, G. Nematicons in Azobenzene Liquid Crystals. Mol. Cryst. Liq. Cryst. 2012, 559, 202-213. [CrossRef]

19. Kohout, M.; Svoboda, J.; Novotná, V.; Pociecha, D. Non-symmetrical bent-shaped liquid crystals based on a laterally substituted naphthalene central core with four ester groups. Liq. Cryst. 2011, 38, 1099-1110. [CrossRef]

20. Kohout, M.; Svoboda, J.; Novotná, V.; Pociecha, D.; Glogarová, M.; Gorecka, E. A nematic-polar columnar phase sequence in new bent-shaped liquid crystals based on a 7-hydroxynaphthalene-2-carboxylic acid core. J. Mater. Chem. 2009, 19, 3153-3160. [CrossRef]

21. Trišović, N.; Matović, L.; Tóth-Katona, T.; Saha, R.; Jákli, A. Mesomorphism of novel stilbene-based bent-core liquid crystals. Liq. Cryst. 2021, 48, 1054-1064. [CrossRef]

22. Tschierske, C.; Photinos, D.J. Biaxial nematic phases. J. Mater. Chem. 2010, 20, 4263-4294. [CrossRef]

23. Vanakaras, A.G.; Photinos, D.J. Thermotropic biaxial nematic liquid crystals: Spontaneous or field stabilized? J. Chem. Phys. 2008, 128, 154512. [CrossRef]

24. Peroukidis, S.D.; Karahaliou, P.K.; Vanakaras, A.G.; Photinos, D.J. Biaxial nematics: Symmetries, order domains and field-induced phase transitions. Liq. Cryst. 2009, 36, 727-737. [CrossRef]

25. Madsen, L.A.; Dingemans, T.J.; Nakata, M.; Samulski, E.T. Thermotropic Biaxial Nematic Liquid Crystals. Phys. Rev. Lett. 2004, 92, 145505. [CrossRef] [PubMed]

26. Acharya, B.R.; Primak, A.; Kumar, S. Biaxial Nematic Phase in Bent-Core Thermotropic Mesogens. Phys. Rev. Lett. 2004, 92, 145506. [CrossRef] [PubMed]

27. Harden, J.; Mbanga, B.; Éber, N.; Fodor-Csorba, K.; Sprunt, S.; Gleeson, J.T.; Jákli, A. Giant Flexoelectricity of Bent-Core Nematic Liquid Crystals. Phys. Rev. Lett. 2006, 97, 157802. [CrossRef] [PubMed]

28. Kapanowski, A. Flexoelectric Effect in Biaxial Nematics. Acta Phys. Pol. A 2011, 122. [CrossRef] 
29. Jákli, A. Liquid crystals of the twenty-first century-Nematic phase of bent-core molecules. Liq. Cryst. Rev. 2013, 1, 65-82. [CrossRef]

30. Jákli, A.; Lavrentovich, O.D.; Selinger, J.V. Physics of liquid crystals of bent-shaped molecules. Rev. Mod. Phys. 2018, 90, 045004. [CrossRef]

31. Matharu, A.S.; Jeeva, S.; Ramanujam, P.S. Liquid crystals for holographic optical data storage. Chem. Soc. Rev. 2007, 36, 1868-1880 [CrossRef]

32. Sobolewska, A.; Bartkiewicz, S.; Mysliwiec, J.; Singer, K.D. Holographic memory devices based on a single-component phototropic liquid crystal. J. Mater. Chem. C 2014, 2, 1409-1412. [CrossRef]

33. Gibbons, W.M.; Kosa, T.; Palffy-Muhoray, P.; Shannon, P.J.; Sun, S.T. Continuous grey-scale image storage using optically aligned nematic liquid crystals. Nature 1995, 377, 43-46. [CrossRef]

34. McPhail, D.; Gu, M. Use of polarization sensitivity for three-dimensional optical data storage in polymer dispersed liquid crystals under two-photon illumination. Appl. Phys. Lett. 2002, 81, 1160-1162. [CrossRef]

35. Kohout, M.; Kozmík, V.; Slabochová, M.; Tůma, J.; Svoboda, J.; Novotná, V.; Pociecha, D. Bent-shaped liquid crystals based on 4-substituted 3-hydroxybenzoic acid central core. Liq. Cryst. 2015, 42, 87-103. [CrossRef]

36. Bisoyi, H.K.; Li, Q. Light-Driven Liquid Crystalline Materials: From Photo-Induced Phase Transitions and Property Modulations to Applications. Chem. Rev. 2016, 116, 15089-15166. [CrossRef] [PubMed]

37. Delden, R.A.v.; Mecca, T.; Rosini, C.; Feringa, B.L. A Chiroptical Molecular Switch with Distinct Chiral and Photochromic Entities and Its Application in Optical Switching of a Cholesteric Liquid Crystal. Chem. Eur. J. 2004, 10, 61-70. [CrossRef]

38. Moran, M.J.; Magrini, M.; Walba, D.M.; Aprahamian, I. Driving a Liquid Crystal Phase Transition Using a Photochromic Hydrazone. J. Am. Chem. Soc. 2018, 140, 13623-13627. [CrossRef] [PubMed]

39. Blanke, M.; Balszuweit, J.; Saccone, M.; Wölper, C.; Doblas Jiménez, D.; Mezger, M.; Voskuhl, J.; Giese, M. Photo-switching and -cyclisation of hydrogen bonded liquid crystals based on resveratrol. Chem. Commun. 2020, 56, 1105-1108. [CrossRef]

40. Bobrovsky, A.; Ryabchun, A.; Cigl, M.; Hamplová, V.; Kašpar, M.; Hampl, F.; Shibaev, V. New azobenzene-based chiralphotochromic substances with thermally stable Z-isomers and their use for the induction of a cholesteric mesophase with a phototunable helix pitch. J. Mater. Chem. C 2014, 2, 8622-8629. [CrossRef]

41. Bobrovsky, A.; Shibaev, V.; Cigl, M.; Hamplová, V.; Hampl, F.; Elyashevitch, G. Photochromic LC-polymer composites containing azobenzene chromophores with thermally stable Z-isomers. J. Mater. Chem. C 2014, 2, 4482-4489. [CrossRef] 